\title{
RACONTER POUR TÉMOIGNER : LA GUERRE ET L'OCCUPATION DANS LE CHAMP LITTÉRAIRE BELGE AU SORTIR DU SECOND CONFLIT MONDIAL
}

\begin{abstract}
Teklik Joanna, Raconter pour témoigner : la guerre et l'Occupation dans le champ littéraire belge au sortir du second conflit mondial [To narrate in order to give evidence : the war and the occupation in the Belgian literature of a direct Post-War period], Studia Romanica Posnaniensia, Adam Mickiewicz University Press, Poznań, vol. XXXIX/1: 2012, pp. 59-67, ISBN 978-83-232-2410-5, ISSN 0137-2475, eISSN 2084-4158.

Over the period directly following the Second World War, continuity and discontinuity were closely interwoven in the Walloon literature. From one hand, esthetic models from Interwar period were still present and no novel concepts appeared in the writings of Walloon authors. From the other, however, a large number of new State-run institutions were inaugurated. The hardships suffered during the war, seen mainly as an instance of disruption, are abundantly described in literary works, especially in testimonies. It is in this context that Arthur Haulot, Belgian poet and novelist, made prisoner and taken to Dachau, created his literary poignant output. His writings are marked by harsh experiences undergone in camps.
\end{abstract}

Keywords. Belgium, World War Two, concentration camp, literary testimonies, Arthur Haulot

Quand on feuillette les ouvrages consacrés à l'histoire des lettres belges, on s'aperçoit que la période de l'après-guerre est l'une des plus méconnues et parfois même sous-estimées par les spécialistes. Rares sont les études exclusivement consacrées à la vie littéraire à la Libération, conçue dans toute sa complexité, et peu nombreux sont les chercheurs qui focalisent leurs études sur cette période ${ }^{1}$. Cela résulte, en partie, du retard en matière de l'histoire de la Seconde Guerre en Belgique qui tient généralement à quelque blocage, intimement lié au dénouement de la Question royale ${ }^{2}$. Pour des raisons évidentes, le monde politique préfère que les archives et les témoignages soient cadenassés et certaines affaires classées sans suite. Les sujets liés

${ }^{1}$ Les travaux de Marc Quaghebeur, ainsi que ceux de Bibiane Fréché dont il sera question plus loin y échappent, en proposant une analyse pertinente de la situation culturelle en Belgique francophone de l'après-guerre.

${ }^{2}$ Le problème de la capitulation de Léopold III engendre dans l'après-guerre un divorce politique irréversible en Belgique, puisque lors du référendum de 1950, les citoyens se divisent en majorité flamande pour le retour du roi sur son trône et en majorité wallonne en faveur de son abdication. Jean Duvieusart, Premier Ministre lors des faits, est l'un des premiers à décrire, en tant que témoin, la Question royale, ainsi que ces conséquences pour la société belge. $C f$. J. Duvieusart (1976). 
à l'Occupation et les années de guerre, tels les rapports avec Hitler ou la collaboration et la répression de l'incivisme, sont devenus délicats, vu l'attitude réelle ou supposée de Léopold III. Il a fallu attendre la fin des années soixante seulement pour que les souvenirs des acteurs de la période 1940-1944 voient le jour. En 1968, les anciens ministres décident de porter un témoignage dans une série télévisée Télémémoires qui provoque un choc dans l'opinion publique. Parallèlement sont menées des recherches, sous l'égide d'un centre qui publie une série d'études consacrées à la déportation, la résistance, la presse clandestine, ainsi qu'à la politique étrangère de la Belgique ${ }^{3}$. Les résultats ne se font pas attendre. En 1971 paraît l'ouvrage de José Gotovich et de Jules-Gérard Libois, L'an 40, la Belgique occupée qui remporte un succès éditorial aussi grand qu'inattendu et légitime désormais l'approche scientifique de la période en question. Le travail, enrichi grâce à l'accès aux archives privées, ainsi qu'aux fonds allemands, élucide et met dans une lumière nouvelle l'histoire des rapports entre Léopold III et Hitler'.

Les recherches menées dans les domaines politique, historique et économique ne favorisent pourtant pas les études parallèles sur les activités culturelles en Belgique pendant et après la Seconde Guerre mondiale. Cette lacune relative est d'autant plus importante que la situation de la littérature belge de l'après-guerre peut être considérée, pour maintes raisons, comme contradictoire. Bien que l'Occupation, tout paradoxal que cela paraisse, offre aux deux parties du pays une occasion inattendue d'accélérer leur propre émancipation (la Flandre se libère de la domination des cultures française et anglaise et la Wallonie se libère du joug de la culture française), ici et là on entend toujours l'écho du Manifeste du Lundi, considéré comme « la Bible idéologique du discours littéraire de l'après-guerre » alors qu'on assiste à la singularisation du champ littéraire belge par rapport à son homologue français (Quaghebeur, 1997 : 236). D’une part, la structure littéraire de l'après-guerre belge francophone reste sous l'influence de la période précédente et la Libération confirme l'intérêt pour le classicisme propre aux années 30 et même à l'Occupation. Le néoclassicisme se fraie le chemin et cherche sa place dans la vie littéraire de l'après-guerre. Il se caractérise, pour reprendre les mots de Bibiane Fréché, par « un souci exacerbé de la perfection formelle », l'intemporalité universelle et le retour à la vision abstraite de l'homme (Fréché, 2009 : 150-151). Les néoclassiques refusent tout engagement et optent pour une écriture anhistorique, à caractère universel, en refusant tout rapprochement avec l'actualité. D'autre part, des dispositifs institutionnels nouveaux sont mis en place et on adopte de nouvelles voies de développement, avec l'appui important de l'État. Ceci étant, les partisans de

3 Je pense en l'occurrence au Centre national des deux Guerres mondiales (1967) qui publie Cahiers d'histoire de la Deuxième Guerre mondiale.

${ }^{4}$ La plupart des sources relatives à la Seconde Guerre mondiale ont été publiées vers la fin du $\mathrm{XX}^{\mathrm{e}}$ siècle et Le Dictionnaire de la Seconde Guerre mondiale en Belgique, paru récemment sous la direction de P. Aron et de J. Gotovitch (2008) fait non seulement le bilan des recherches mais aussi permet de mieux comprendre cette période particulièrement sensible de l'histoire de Belgique. 
la continuité ou de la discontinuité de la littérature belge de l'époque ont, chacun, dans un certain sens, raison ${ }^{5}$.

$\mathrm{Au}$ sortir de la Seconde Guerre mondiale, le champ littéraire belge connaît un moment "d'effervescence créatrice » presque inégalé depuis sa naissance (Quaghebeur, 1980 : 505). Même si cette vitalité a un caractère éphémère et que l'allégresse ressentie en septembre 1944 se fasse bientôt oublier, un nombre important d'écrivains, éditeurs et critiques reprennent et réhabilitent la vie littéraire. Malgré les difficultés matérielles, on observe la floraison des revues (nonante et une revues durant les cinq années qui suivent la Libération) et des prix littéraires qui s'inscrivent dans cette ambiance générale (plus de trente-cinq prix sont institués après la Libération) (Fréché, 2009 : 9). Paradoxalement donc, la littérature belge soumise au contrôle exercé par l'occupant, s'autonomise par rapport à la littérature française. De nouvelles plumes émergent en Belgique, des salles de spectacle et des maisons d'édition plus indépendantes du centre parisien voient alors le jour. Le plus grand bénéficiaire de cette situation est le théâtre qui s'institutionnalise et se professionnalise avec le temps. Un nouveau système de subventionnement public, ne doit pas passer inaperçu, de même que l'émergence de certains noms devenus bientôt phares de la littérature belge (Charles Bertin, Michel de Ghelderode, Suzanne Lilar, Paul Willems, etc.). Durant cette période qui s'étend jusqu'aux années cinquante, les livres d'auteurs belges se vendent bien, vu la politique d'achat qui renforce leur position dans le pays. À cela s'ajoute une réforme des Beaux-Arts et des Lettres, réalisée par le Ministère de l'Instruction publique, qui aboutit à la création du Théâtre National et celle du Fonds national de la littérature, subventionnant d'ailleurs l'édition littéraire locale.

Or, malgré tout ceci, l'Occupation pèse de toutes ses contraintes dans tous les domaines de la vie de l'après-guerre, y compris la littérature. La reconstruction ne débouche pas sur une vision univoque du passé ce qui empêche de dresser un bilan nettement positif. L'un des obstacles principaux réside dans la question de la collaboration des intellectuels. Afin de pouvoir en juger, il faudrait commencer par distinguer de ce qu'on appelle l'activité inconditionnelle au profit de l'occupant (la collaboration proprement dite) et l'accommodation qui, sur le terrain belge, désigne la poursuite de l'activité dépourvue d'accents ouvertement pro-nazis. Outre la collaboration et l'accommodation (donc la collaboration involontaire), certains intellectuels choisissent la voie du silence - le refus radical de publication quelconque ou la résistance, au sens propre du terme, entendue comme toute publication visant à compromettre l'occupant. Cette complexité de réactions envisageables démontre fort bien la difficulté de jugement et empêche de faire une distinction nette, au lendemain de la guerre, entre la littérature idéologiquement « correcte » et « incorrecte », la

${ }^{5}$ Le problème est largement discuté lors du colloque organisé en 1995 à Bruxelles (Société, culture et mentalité. L'impact de la Seconde Guerre mondiale en Belgique) dont les intervenants, issus des deux côtés de la frontière linguistique, soulèvent à maintes reprises la complexité. $C f$. A. Van Den Braembussche (1997 : 271-274). 
bonne et la mauvaise. À cela s'ajoute la question de l'épuration qui, en Belgique, n'a quasiment pas lieu. Ceux qui se taisent et refusent ouvertement de publier, ne sont jamais récompensés par l'État et ceux qui collaborent ne sont pas toujours punis pour leurs actes. Le problème d'épuration, vu son caractère complexe, fut souvent minimisé au cours des années et ceci des deux côtés des frontières linguistiques. Que ce soit Wallonie ou Flandre, la question de la collaboration reste toujours un sujet controversé.

Par ailleurs, la Belgique n'est pas le seul pays à avoir affronté ce problème. En France ou en Italie, la Libération fut également associée au problème de l'épuration jugée incomplète, quoique réalisée dans un contexte différent. Du côté belge, on ne trouverait pas cette initiative institutionnelle présente chez le voisin français et visant à réorganiser la vie culturelle du pays à travers la mission confiée aux intellectuels durant la Résistance. On ne trouverait pas non plus de nouvelles structures littéraires puisqu'il n'y a pas une vraie rupture d'avec l'entre-deux-guerres. La Résistance n'a pas le caractère structurel et elle ne donne pas naissance à un corpus littéraire aussi important que représentatif pour la période en question. Les auteurs belges publient en contournant la censure allemande jusqu'en 1943 et la période de l'après-guerre n'est pas marquée par les débats déclenchés autour du problème de la collaboration. Ainsi la Libération en Belgique s'inscrit dans la continuité pas rapport à la Première Guerre mondiale qui, quand à elle, constitue une vraie fracture dans la périodisation de la littérature belge et fait naître la littérature patriotique qu'on chercherait en vain dans la Belgique francophone de 1945. À cela s'ajoute le fait que les hommes de lettres belges n'ont pas alors la légitimité suffisante pour se prononcer en public sur l'actualité et ils cèdent souvent la place à un chercheur dans le domaine. Aucun écrivain ne répond littérairement en Belgique au roman Châtiment des victimes de José-André Lacour, pas plus qu'on ne réagira au recueil que Paul Dresse publie au plus fort de la Question royale.

Afin de mieux comprendre le rapport entre les lettres belges de l'après-guerre et l'Histoire, il faut regarder de plus près comment cette dernière est convoquée dans la littérature, celle de témoignage en particulier. Passée l'heure de la guerre, certains écrivains belges décident de transcrire leur expérience traumatisante, la vie quotidienne sous l'Occupation, les camps et la Résistance. À croire les statistiques, durant les années de l'après-guerre en Belgique voient respectivement le jour quarante-sept romans, trente-quatre récits courts, quatre-vingt-huit recueils poétiques, onze pièces de théâtre et plusieurs témoignages, considérés par la critique comme littéraires (Fréché, 2009 : 237) ${ }^{6}$. Parmi eux, celui d'Arthur Haulot (1913-2005) qui fait autorité en matière et reste l'un des interprètes les plus connus de ce vécu singulier en Belgique de l'après-guerre. Il revient en 1945 avec son témoignage sur Dachau et il est le seul à émerger comme poète (deux recueils, Si lourd de sang et D'entre les morts de

${ }^{6}$ Les chiffres en question valent pour la période allant de septembre 1944 à fin 1951, durant laquelle se condensent la majorité des publications liées directement au traumatisme du conflit. 
1947)7. S'il jouit aujourd'hui de cette reconnaissance symbolique et officielle, ce n'est pas seulement grâce à ses écrits, mais aussi, ou surtout, à sa position publique, ainsi qu'à ses nombreuses fonctions: Président de l'Association internationale des anciens prisonniers de Dachau, Commissaire général au Tourisme, Président du « groupe mémoire », regroupant les anciens combattants et les ex-prisonniers politiques, militant actif contre l'extrême-droite, animateur littéraire connu, veillant jusqu'à la fin de sa vie à l'animation de la Biennale de la poésie.

Haulot-témoin émerge dans l'immédiat après-guerre en tant qu'écrivain, mais au moment où il prend part au débat portant sur la Question royale, il se prononce en qualité d'ancien prisonnier. C'est ainsi qu'en juillet 1945, dans les colonnes d'Alerte, il s'adresse au roi Léopold III en regrettant que le pays soit déchiré par ladite Question qui occupe plus l'opinion publique que les problèmes liés aux conséquences de la guerre, tels l'avenir des orphelins, des veuves ou des jeunes. Pour lui, le souverain avec ses conseillers insultent la souffrance de ceux qui ont vécu l'horreur des fours crématoires (Haulot, 1945 : 3). Son discours en faveur de l'effacement volontaire du roi se fait entendre beaucoup mieux cinq ans plus tard où la CNPPA (la Confédération nationale des prisonniers politiques et ayants droit) dont il est membre, jusqu'alors neutre en la matière, décide de prendre position et d'intervenir afin d'obtenir l'abdication du roi, considérée comme la solution la plus sage car garantissant à la Belgique l'équilibre politique et social. Arthur Haulot devient désormais une autorité qui se prononce sur les questions d'actualité. Toutefois, son activité traduit une fois de plus les paradoxes présents dans la vie littéraire de l'après-guerre belge. Bien qu'il soit engagé, en tant que membre de l'Association socialiste des écrivains et artistes, son engagement ne peut pas être conçu au sens sartrien du terme. L'Association manque de contact avec les cadres politiques et aucune synergie ne lie les intellectuels et le parti socialiste. Contrairement à ce qui se passe alors en Italie où une nouvelle éthique s'établit, tout en contribuant au développement de l'esthétique néo-réaliste ${ }^{8}$, la gauche littéraire belge est incapable d'agir sur la politique culturelle du parti et, encore moins, sur la vie littéraire. Même si l'écrivain est appelé à s'engager, on le laisse libre sur ses choix esthétiques ( $c f$. « Pour la défense de la culture », Le Peuple, $1956: 6$; Fréché, 2009 : 284-85).

Les paradoxes mis à part, le témoignage poignant d'Haulots'inscritindubitablement dans ce genre de littérature qui ne laisse pas le lecteur indifférent. Poète passionné,

7 Ceci est intimement lié au rôle qu'il joue dans l'organisation des Biennales internationales de poésie de Knokke. Depuis 1951, une équipe de « Journal des poètes », réunie autour de P.-L. Flouquet et de A. Haulot, contribue à la création d'un réseau poétique international et, par là, à l'intégration des poètes belges dans le champ français.

8 À travers diverses polémiques, publiées dans la presse, les intellectuels italiens s'interrogent sur le rôle qu'ils jouent ou devraient jouer dans la société et cherchent à définir le rôle et la responsabilité de la culture face à la politique. En renouant avec la conception gramscienne de la littérature nationale-populaire, ils indiquent une voie nouvelle à prendre, celle du réalisme créé avec l'esprit de l'après-coup et enraciné fortement dans le présent sociopolitique. 
humaniste acharné, militant socialiste, pionnier du tourisme pour tous, il reste avant tout le rescapé de Dachau, témoin de l'horreur vécue qu'il transcrit dans sa poésie. Arrêté par la Gestapo à la fin de 1941, envoyé au camp de Mauthausen en catégorie N.N., il arrive pourtant, après son transfert à Dachau, en 1942, à échapper à la mort. Tout au long de sa détention, il fait preuve de la résistance en clandestinité, organise le comité international et n'hésite pas à prendre le commandement du camp au moment de sa libération par l'armée américaine. Ces derniers événements sont soigneusement relatés dans le texte qu'il rédige entre mai et juin 1945, avec son camarade albanais, Ali Kuci. Leur récit constitue la première partie du témoignage au titre évocateur Dachau où il se réfère aux huit derniers jours du camp en question. La seconde partie, écrite par Haulot seul à la même période, retrace son itinéraire « aux limites du monde », comme l'appelle l'auteur, celui qui le conduit, de manière chronologique, de Mauthausen vers Dachau. Jour par jour, heure par heure, le « je » du narrateur, dilué souvent en « nous » désignant l'ensemble des détenus raconte de façon minutieuse la réalité concentrationnaire, réduite à l'attente désespérée des libérateurs. À côté des informations et données purement statistiques (histoire de Dachau, nombre de détenus, estimé selon la saison ou l'année, épidémie déclarée, arrivée ou départ des prisonniers), le récit est imprégné de souvenirs frappant à tout moment l'imagination du lecteur. Les rares photos qui accompagnent le texte, prises probablement au moment de la libération de Dachau, ne font qu'accroître cette impression. Les éléments propres à la réalité concentrationnaire surgissent de ces photos et il est impossible d'oublier le spectacle atroce qu'offre par exemple un amoncellement de cadavres dans la chambre à gaz de Dachau (Haulot, Kuci, 1945 : 86).

Un désespoir profond marque le quotidien des détenus, hommes désaxés, arrachés à la vie normale, à la morale, à la famille, au groupe humain, laissés en tête à tête avec les instincts les plus bas et les tentations les plus tristes, les plus déshonorantes (p. 24-25) ${ }^{9}$. L'auteur déploie sous les yeux du lecteur l'aspect épouvantable du camp, avec phrases saccadées, courtes et riches en points d'exclamations. Non sans raison, on trouve dans le témoignage d'Haulot des références à l'enfer dantesque (p. 95, 125), associées souvent aujourd'hui à l'écriture de Primo Levi. La seconde partie de Dachau est d'ailleurs évocatrice à ce titre. L'itinéraire aux limites du monde, proposé au lecteur, reprend le schéma habituel du témoignage portant sur le passé concentrationnaire. De façon chronologique, chapitre par chapitre, on suit les étapes consécutives de la déportation : le choc de l'arrivée et l'adaptation à la nouvelle réalité (p. 93-103), l'épuisement physique lié au travail (p.104-106), la faim (p. 109-111), la souffrance, l'avilissement, enfin la mort omniprésente (p. 107-108), et ceci au rythme saccadé des ordres des SS:

Vite, à poil, tout le monde. Vite, à l'inscription, Noms, prénoms. Une fiche, un numéro. Vous avez fini d'être un être humain. Vite, plus vite, sur le ciment brûlant, vers le bain, dans la cave. Un homme à torse nu vous happe, vous tend la tête, le pubis, les aisselles. Un autre vous ba-

\footnotetext{
${ }^{9}$ La pagination renvoie à l'édition mentionnée dans la bibliographie.
} 
digeonne à grands coups de pinceau trempé dans le pétrole. D'autres sont aux manettes des douches. Tempo ! Tempo ! [...] De l'eau glacée, d'abord. Puis, la voici bouillante. On hurle dans la vapeur [...]. Stop (p. 97-98).

Une telle scène se reproduit d'un témoignage à l'autre, même si les acteurs changent parfois. À maints égards, le témoignage d'Arthur Haulot rappelle celui de David Rousset, militant trotskiste français, déporté à Buchenwald qui publie son Univers concentrationnaire en 1946. Chez Rousset, l'entrée du détenu dans le monde du camp s'effectue également à un rythme rapide, ce qui est mis en relief par des phrases concises, souvent sans verbes, rappelant en quelque sorte une liste de faits (obligatoirement) accomplis. Il décompose ainsi l'image de l'univers concentrationnaire en morceaux qui préparent l'homme à affronter la nouvelle réalité (Rousset, 1946 : 14-15). Rousset, de même que Haulot, oscille entre les faits et la narration subjective et même s'il cherche à rester objectif dans la transcription de son vécu, s'efforçant souvent à une simple description des faits et réduisant au minimum le commentaire, il laisse pourtant voir des traces latentes de son implication personnelle. À cela s'ajoute le fait que les deux rescapés-témoins ne cessent de mettre en relief la nécessité de lutter contre l'oppression, ce qui est lié à la raison commune, politique, de leur détention (Rousset, 1946 : 75). Les deux enfin, tout en présentant la réalité où les valeurs éthiques et esthétiques sont contestées et la loi violée maintes fois, garantissent l'authenticité de leur témoignage, en rappelant sans cesse que tout est possible (Rousset, $1946: 181$ ) dans l'univers des camps et que l'homme n'en sort jamais indemne : Tant pis si des âmes sensibles disent: " ce n'est pas vrai » ou «ce n'est pas possible ». Cela est! (p. 110).

Le témoignage de Arthur Haulot suit le chemin de tant d'autres, que ce soit du côté français, italien ou polonais. L'avant-propos à Dachau en est révélateur. Bien que son texte soit rédigé quasi immédiatement après la guerre, en juin 1945, il se soucie de l'impact de son témoignage, tout en parlant du public qui se lasse déjà, mais aussi frisonne de dégouts à la révélation des charniers de Dachau, Buchenwald, Mauthausen, Dora.... (p. 7-8). Il traduit ainsi les doutes des autres survivants qui craignent, à leur retour, qu'on refuse de croire, à tant d'atrocités, à tant de science dans l'assassinat, à tant de raffinement dans le massacre (p. 8). Jean Cayrol, déporté à Mauthausen, rejoint cette idée et va plus loin, en excluant toute autre forme que témoignage. Puisque, comme il dit, l'expérience concentrationnaire est intransmissible, solitaire, instable; elle se vit ou se meurt (Cayrol, 1953 : 576). Haulot y souscrit en observant qu'il faut que " ceux de camps » qui le peuvent soient leurs propres témoins, qu'ils parlent, qu'on les entende (p. 9). Et ceci pour garantir toujours l'authenticité et la sincérité du témoignage sur le vécu aussi grave que singulier, afin qu'il ne se reproduise plus.

Enquêté en 1996 par l'Association «Territoires de la Mémoire », à l'occasion de son parrainage, Haulot reconnaît que, contrairement à beaucoup de rescapés des camps, qui ont préféré faire le silence sur leur indicible expérience, il a toujours pensé qu'il fallait que les gens sachent de quel prix avaient été payés la démocratie, la sécurité 
et le bien-être dont ils jouissent aujourd'hui. Il est, dit-il, essentiel, à mon sens, de perpétuer le savoir, d'informer les jeunes, non seulement pour rendre un hommage légitime à ceux qui ont fait tous les sacrifices pour restaurer les valeurs essentielles, mais aussi pour faire prendre conscience qu'ils ne sont pas nécessairement à l'abri de ce qui est arrivé à leurs parents et à leurs grands-parents ${ }^{10}$. Son témoignage Dachau se clôt d'ailleurs par l'hommage rendu aux témoins intégraux, donc ceux qui sont morts dans les camps (cf. P. Levi). Quoi qu'il fasse, dit Haulot, qu'il se souvienne ou qu'il oublie, le monde de demain sera marqué par vous (Haulot, 1945 : 170)

L'exemple du témoignage remarquable d'Arthur Haulot (sa poésie, pour des raisons d'économie, n'entre pas dans le cadre du présent travail) démontre fort bien la singularité de l'expérience concentrationnaire, commune à tous les rescapés, quelle que soit leur nationalité ou origine sociale. Cela n'empêche pourtant pas de constater que les auteurs issus de la Seconde guerre mondiale ne se constituent pas en groupe et que la littérature de témoignage ne connaît aucune postériorité significative. Son infortune vient non seulement de la carence des structures littéraires d'accueil, mais aussi des choix esthétiques adoptés. La plupart des auteurs de textes sur la guerre certifient la véracité et la connaissance des faits qu'ils rapportent et dénient toute recherche esthétique. Les préfaces abondent en indications qui ôtent toute compétence littéraire à l'auteur, et ceci afin de rassurer le lecteur de l'authenticité du texte et de la sincérité de son auteur. Comme si la véracité historique ne pouvait être compatible avec vraisemblance littéraire (Grierson, 2003 : 125-131). Dans les années cinquante seulement, sont publiés quelques livres plus aboutis, semble-t-il, vu la distance que prennent les auteurs afin de se distancier par rapport à l'événement. Ils annoncent la seconde phase de la littérature de témoignage où la guerre, comme l'observe Marc Quaghebeur, sert plutôt de base contextuelle que de thème central (Quaghebeur, 1997 : 269). La première vague est constituée par des livres et/ou témoignages, publiés quasi immédiatement après la guerre, donc dans les années 40-50. À ce groupe appartiennent entre autres les ouvrages de David Scheinert et de Daniel Gillès, Châtiment des victimes de José-André Lacour (1949), Délire logique de Paul Nothomb (1948) ou L'Enfer Ciel de Robert Poulet (1952). La seconde vague ressurgit dans les années septante seulement et marque une nouvelle étape dans la représentation des événements liés à la Seconde Guerre mondiale qui, désormais, est présentée de façon plus approfondie et à la lumière des recherches récentes. À côté des noms des écrivains mentionnés ci-dessus, tels Gillès (qui inaugure en 1974 un cycle romanesque intitulé Le Cinquième commandement), Lacour (Rêve de Caïn de 1980) apparaissent d'autres auteurs qui reviennent à la problématique de la guerre. Parmi eux Pierre Mertens, avec ses Eblouissements (1987) ou Une paix royale (1995) qui traite de la Question royale ou Jean Louvet avec L'Homme qui avait le soleil dans sa poche (1980). Or, ces livres ne cherchent plus à présenter explicitement les

${ }^{10} C f$. l'article « Arthur Haulot : le sens d'un parrainage », publié dans « Territoires de la Mémoire $», \mathrm{n}^{\circ} 1$, septembre-novembre 1996. 
événements du passé tragique et traduisent plutôt la mémoire qui fait irruption dans le champ historique. Si l'histoire de la Seconde Guerre mondiale revient sur leurs pages, elle est véhiculée par une écriture spécifique, fragmentaire, métaphorique et réduite souvent au silence évocateur (Quaghebeur, 1997 : 270).

Quelle que soit la date de leur publication, les livres des écrivains touchés par les événements de leur siècle montrent qu'il est difficile de sortir indemne de son passé et de préserver sa sensibilité d'avant. Ils tendent davantage à susciter une réflexion sur le sens même de l'Histoire, sur le rôle de la littérature en ce qui concerne la conscientisation des citoyens, sur une manière d'envisager le monde selon des points de vue diversifiés et sans nul doute plus fraternels, sur les ambiguités du réel dès qu'il est inséré au cœur d'une fiction. J'espère avoir montré, par cette brève incursion dans les méandres de l'histoire littéraire de la Belgique de l'après-guerre, qu'il reste du pain sur la planche pour les spécialistes de littérature comparée et que l'exemple belge invite à être étudié d'une manière plus approfondie, non seulement par rapport à la situation en France, mais aussi par rapport à d'autres pays qui connurent l'expérience de la guerre et de l'Occupation.

\section{BIBLIOGRAPHIE}

Aron, P., Gotovitch, J. (dir.) (2008). Dictionnaire de la Seconde Guerre mondiale en Belgique, Bruxelles : André Versaille éditeur.

Cayrol, J., « Témoignage et littérature », Esprit, avril 1953.

Centre national des deux Guerres mondiales (1967). Cahiers d'histoire de la Deuxième Guerre mondiale, Bruxelles : Pierre De Meyer.

Duvieusart, J. (1976). La Question royale. Crise et dénouement, juin, juillet, août 1950, Bruxelles : CRISP.

Fréché, B. (2009). Littérature et société en Belgique francophone (1944-1960), Bruxelles : Le CRI/ CIEL-ULB-Ulg.

Gérard-Libois, J., Gotovitch, J. (1971). L'an 40. La Belgique occupée, Bruxelles : CRISP.

Grierson, K. (2003). Discours d'Auschwitz. Littérarité, représentation, symbolisation, Paris : H. Champion.

Haulot, A. « Lettre ouverte à Léopold III », Alerte, n 30, 26 juillet 1945.

Haulot, A., Kuci, A. (1945). Dachau, Bruxelles : Éd. Est-Ouest.

Quaghebeur, M. (1997). Éléments pour une étude du champ littéraire belge francophone de l'aprèsguerre in Leurs occupations. L'impact de la Seconde Guerre mondiale sur la littérature en Belgique, Bruxelles : Textyles-CREHGSM.

Quaghebeur, M. (1980). Littérature et fonctionnement idéologique en Belgique francophone in La Belgique malgré tout, Bruxelles : Éditions de 1'Université de Bruxelles.

Rousset, D. (1946). L'univers concentrationnaire, Paris : Éd. du Pavois.

Van Den Braembussche, A. (1997). Littérature et impact de la Seconde Guerre mondiale en Belgique. Essai de synthèse in Leurs occupations. L'impact de la Seconde Guerre mondiale sur la littérature en Belgique, Bruxelles : Textyles-CREHGSM. 